\title{
How Do People's Concepts of Place Relate to Physical Locations?
}

\author{
Changqing Zhou, Pamela Ludford, Dan Frankowski, and Loren Terveen \\ Department of Computer Science and Engineering, \\ University of Minnesota, \\ 200 Union ST SE, 4-192, \\ Minneapolis MN 55414 \\ \{czhou, ludford, dfrankow, terveen\}@cs.umn.edu
}

\begin{abstract}
Advances in GPS and wireless networking technologies have enabled a new class of location-aware applications, including location tracking 102, location-enhanced messaging 39, location-based gaming(www.botfighters.com), and navigation aids for the visually impaired [12. However, these applications typically represent places quite simply, as a geographical point or a point plus radius. We conducted an experiment that showed that this simple representation is not expressive enough to represent the full range of people's everyday places. We also present a set of more complicated physical shapes that our subjects found sufficient to cover their places. These results identify representational requirements for location-aware systems, have implications for systems that aim to acquire place representations, suggest enhanced applications, and open up interesting avenues for future research.
\end{abstract}

\section{Introduction}

Most existing location-aware systems, both research prototypes and commercial applications, represent locations as a point (e.g., a latitude/longitude) or point plus radius (e.g., 50 meters). A typical use of such information is for an application to take some action when a user crosses the "threshold" of a place. For example, GeoNotes [5] will present relevant notes, and uLocate(www.uLocate.com) can notify a "watching" user that a "watched" user has crossed a "geofence".

comMotion [14 took a step beyond the direct, simple relationship of places to physical locations. A comMotion device inferred the existence of a new place when it lost a GPS signal three different times at the same location (the intuition is that this often signaled entering a building, and many places correspond to buildings). When this happened, it presented its guess to the user (on a visual map interface) for labeling. Further, while each place by default was treated as a distinct place (for comMotion, this meant that it received its own "to-do" list), a user could choose to associate the same list with multiple locations; for example, someone might create a single grocery list for all grocery stores. Thus, users could create an entity consisting of distinct physical locations that the application treated somewhat like a single place. 
We believe that the point + radius representation of a place is not always sufficient, and more complicated representations need to be introduced to accomendate places like "all grocery stores". Our first basis for this assertion is simply reflecting on our everyday places. A favorite bike route, any coffee shop in a small city, or even a five-block-long stretch of a street filled with interesting shops and nightclubs might be very meaningful places to a person and his friends. We also note that Geographical Information Systems offer a rich set of geometric representations, such as points, multiple points, and regions [19]. These data structures were developed to represent geographic features such as roads, rivers, and political boundaries. However, we suspect they will be useful for representing people's personal places, too. Finally, we observe that the GIS geometric representations and people's conceptual representations of places may not always agree. In virtual reality research, places are represented both symbolically and literally to give designers the freedom to present important details without worrying about the correct scale, ratio, etc [16]. A person may perceive a large city as a point simply because it is far away and his only memory about the city is the conference center he went to; by contrast, this person may perceive his grandparent's small town in many detailed shapes, such as the local roads, shops and restaurants, the river and streams that flow by, etc.

Research in environmental psychology has shown that people naturally structure their experience around socially meaningful places - home, office, school, church, coffee shop, pub, etc [6]. A number of research projects have taken steps toward discovering such places from location data. As we already have discussed, comMotion 14 was one such effort. Ashbrook and Starner 1 applied a clustering algorithm to group GPS readings into what they called "significant locations". Zhou et al. explicitly sought to discover meaningful places, presented an algorithm for discovering places from location data 21, and conducted an empirical evaluation of their algorithm data 22 . Other researchers have explored the use of methods such as Markov models and Bayesian networks to learn significant locations and probable transitions between them 15]13. Based on the results of an empirical investigation of the actual descriptions people produce in mobile phone conversations, Wilenmann and Leuchovius proposed that locationbased services should describe location in ways relevant to users, such as "I'm home", "Where we met last time", "So you'll be here in five minutes" [20].

If one believes that the concept of place is useful in location-aware systems, then a fundamental research question is:

How do people's concepts of place relate to the representations of physical locations used by computational systems?

We carried out an empirical study to answer this question; this paper reports the results.

The remainder of the paper is organized as follows. We first present some background concepts, then describe our experimental design. Next, we present our results and follow that with a discussion of design implications for locationaware applications. Finally, we close with a brief summary. 


\section{Background Concepts}

In this section, we describe the data collection process and a set of place shapes that we used in our experiment.

\subsection{Sources of Data}

To attempt to answer our question, we needed two types of data: (1) information about people's everyday places, and (2) some computational representation of physical locations that correspond to these places. Details are given in the description of the experimental design below. However, at a high level, we obtained place information by having subjects keep a diary, and we obtained physical location information by giving subjects a GPS device that took frequent location readings. We then applied an algorithm [21] to generate a set of discovered places.

\subsection{Proposed Place Shapes}

We went into the study hypothesizing the existence of four place shapes representing possible physical representations a place could assume. We based these shapes on our intuitions and analysis of data from a pilot study. The four shapes are:

- Dot: the simplest category. A single physical location (or 'dot on a map') such as "my house" or "Mariucci Hockey Arena".

- Multiple Dots(Multi-Dots). One conceptual place consisting of separate physical locations or dots on a map. Prototypical examples are chain stores like Starbucks or Barnes \& Noble or generic places like a gas station or grocery store.

- Region: a geographic region. Prototypical examples include urban neighborhoods ("Soho", "Downtown"), recreational parks and University campuses.

- Path: a sequence of locations, e.g., a commuting route or biking/walking path.

\section{$3 \quad$ Experiment}

Subjects. Our intuition was that people's daily activities, and thus the types of places they visited, would depend on their life stage. For example, we expected 20-year-old college students, 40-year-old working parents, or 60-year-old retirees to frequent different types of places. Therefore, we recruited subjects from across this spectrum.

We recruited 28 subjects, all from a major U.S. metropolitan area. Some live in a core city, some in the suburbs. They used a variety of travel modes, including walking, biking, public transportation, and personal car. Their ages ranged from the early 20 s to late $60 \mathrm{~s}$, with an average in the early $30 \mathrm{~s}$. Twenty were male, 8 female. Three subjects had preschool children, 4 had school aged children, and 
2 had adult children not living with them. The subjects were highly educated, with two thirds having college or advanced degrees. They included 6 college students, 4 engineers, 4 information technology professionals, 4 teachers, a range of other professional and service jobs, as well as several retired people. Clearly our sample is limited and unrepresentative in certain ways. However, we believe it is appropriate since we were conducting formative research intended to better characterize a phenomenom and define more precise, quantitative questions for future study.

Data collection. We equipped subjects with a GPS-enabled cell phone (the Motorola i88s, with service from Nextel) that ran Accutracking software (www. accutracking.com). We set the software to take a GPS reading every minute and send it to a server for storage. Subjects carried the phone for about three weeks, and were instructed to keep the phone with them and turned on at all times. However, they could turn off the phone for privacy reasons whenever they wanted. Subjects also kept a diary 818 of the places they visited each day. They received a daily reminder (email, instant message or phone call); they could return their list of places via email or record it in a notebook. We met with all subjects at the beginning of the study to give them the phone, demonstrate its use, and instruct them in the data collection procedure. After data collection was complete, we conducted a semi-structured interview with each subject.

Interview preparation. We ran our discovery algorithm on each subject's personal location dataset (i.e., the GPS readings). For the purposes of this study, the key result of this was that it established a potential correspondence between users' places (as recorded in their diaries) and physical locations. For each subject, we printed out an overview map showing all the discovered places and a set of more detailed maps showing nearby groups of discovered places at a higher level of resolution (see Fig 4 and Fig 5). We also printed a table of each subject's places, shown in Table [1. Note that [1 is filled out to represent the data collected during the interview; initially, the "Shape" and "Comments" columns are blank).

Table 1. Excerpt from the interview table, showing data from one subject

\begin{tabular}{|l|l|l|}
\hline Description & Shape & Comments \\
\hline Home & Dot & \\
\hline Cub Foods & Dot & Where I work \\
\hline TCF Bank & Dot & \\
\hline ME Building & Dot & Where I study \\
\hline Neighbor's house & Dot & \\
\hline Bus stop & Multi-Dots & I ride bus to work and school \\
\hline Parking lot & Multi-Dots & $\begin{array}{l}\text { Many places where I catch a ride from } \\
\text { friends }\end{array}$ \\
\hline Walk to bus stop & Path & I walk 3-4 blocks \\
\hline Rosedale mall & Region & This is where I shop \\
\hline
\end{tabular}


Interviews. We conducted a semi-structured interview with each subject organized around the maps and table. We first led subjects through the process of matching their places (in the table) to the discovered places (on the map). Any discovered places that were not in the diary were added to the table. We then stepped through each place with each subject, asking them to tell us the physical shape of the place. While we presented our shape categories - Dot, Multi-Dots, Region, Path, Other - we also gave subjects the chance to describe places as having a different shape (which we categorized as Other). However, subjects scarcely exercised this option. We also kept notes of the subjects' comments.

\section{Results}

\subsection{The Dataset}

All 28 subjects logged their data for three weeks. Time constraints kept us from scheduling interviews for 3 subjects, so we discarded their data. Also, the interview with one subject was unsuccessful, so we discarded this data as well. Thus, we ended up with data for 24 subjects.

Table 2 summarizes the location readings collected from the subjects. This is a large amount of real data. We know of no other studies with samples of this size. Subjects followed the data collection procedure quite faithfully. The average number of readings per subject is 6,364, which represents over 100 hours of data. Fig. 1]illustrates a subject's GPS data in a metropolitan area collected in a period of 3 weeks.

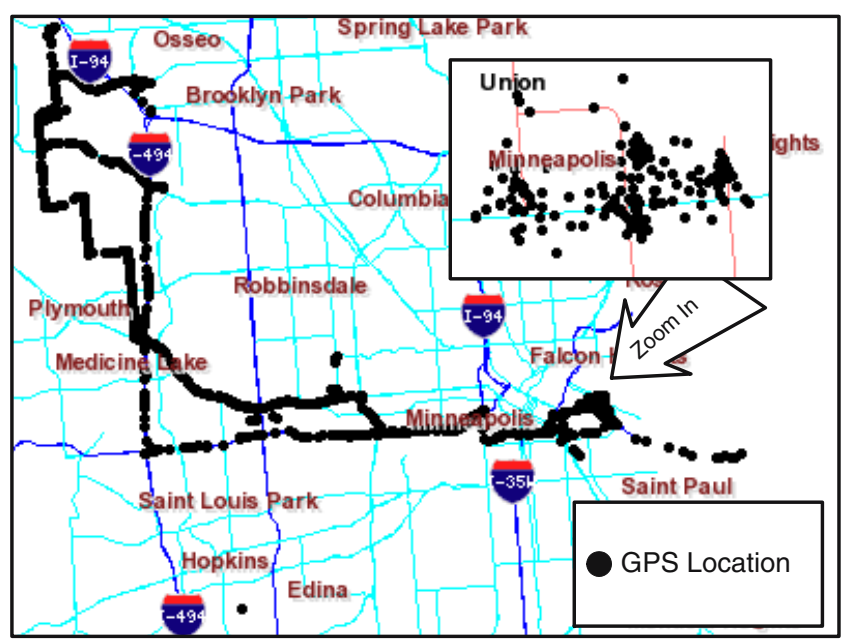

Fig. 1. One subject's GPS data in a metropolitan area collected in a period of 3 weeks. Each dot represents a GPS reading and most of the dots are distributed along major highways. The zoom-in section represents readings on a university campus, which contains 4 places where density of the dots is high. 
Table 2. Personal location dataset: on average, 4.8 hours wirth of GPS data was tracked for each subject

\begin{tabular}{|l|l|l|l|}
\hline Statistics & Readings & Data collect days & Mean readings per day \\
\hline Total & 152,741 & 516 & 7,008 \\
\hline Mean per subject & 6,364 & 22 & 292 \\
\hline Std Dev & 3,997 & 3 & 167 \\
\hline
\end{tabular}

\subsection{Occurrences of Different Place Shapes}

Figure 2(a) summarizes the number of places that fell into each place shape. A large majority $(79 \%)$ of the places were simple locations, or "dots". This may reflect the fact that people typically spend most of their time at relatively fixed and small locations, such as home, office, restaurant, etc. However, $21 \%$ were of more complicated shapes. Figure 2(b) gives the average numbers of places with different shapes: on average each subject has 25 Dot type places, 4 Multi-Dots, 2 Region and 1 Path.

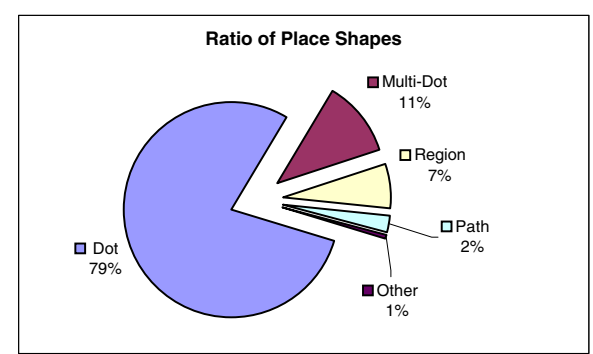

(a)Ratio of different shapes

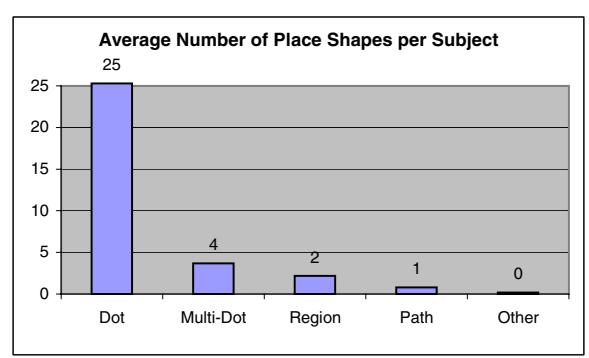

(b)Average number of shapes per subject

Fig. 2. Occurrences of different place shapes: Figure(a) shows that $79 \%$ of the places are Dot type, while $21 \%$ of the places are not; Figure(b) shows that on average, each subject has 7 places that are either Multi-Dots, Region or Path

\subsection{Occurrences of Subjects with Different Place Shapes}

Fig 3(a) shows the number of subjects that had places of each of the different shapes. Again, not surprisingly, every subject has place shape Dot. Although the complicated shapes account for only $21 \%$ of the total shapes, 20 out of 24 subjects have Multi-Dot, 18 have Region and 13 have Path. Fig 3.(b) illustrates the percentage of subjects with different number of shapes: $38 \%$ of the subjects have 3 shapes, $33 \%$ have 4 shapes, $21 \%$ have 2 shapes and only $4 \%$ have 1 shape.

These results suggest that people's everyday places do take on physical shapes that are more complex than simply a point or point + radius. 


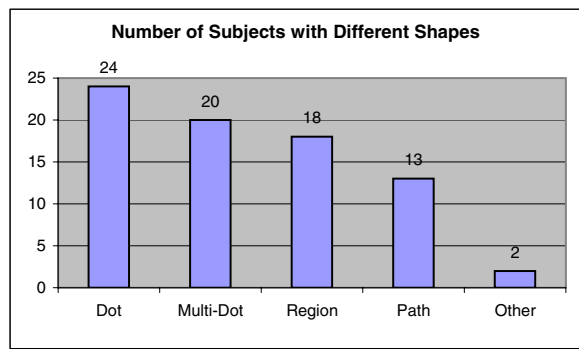

(a)Number of subjects with different place shapes

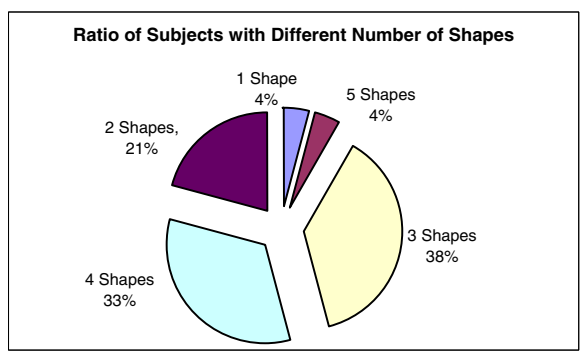

(b)Ratio of subjects with different number of shapes

Fig. 3. Number of subjects with different place shapes: Figure(a) shows more than two thirds of the subjects have Multiple Dot and Region type, and half of them have Path shape; Figure(b) shows that $71 \%$ of the subjects have 3 or 4 different types of place shapes

\subsection{Examining the Place Shapes}

Dot. This type typically represents an atomic geometry, such as home, work, a restaurant, a post office, a friend's house, a parking ramp, a store, a theater. Fig. 4(a) shows 2 places of Dot typs: a subject's home and her friend's house. However, some subjects classified places as Dots that were not "point" geometries. We discuss these situations in the following sections.

Multiple Dots. Subjects found it easy to apply the Multiple Dots category; they commonly placed chain stores (McDonalds, Target, Cub Foods) and generic places (restaurant, gas station, bank) in this category. They would note that, for many purposes, one physical location was as good as another: the same groceris could be bought at any Cub Foods store, for example. However, when some subjects always went to just one particular McDonalds, they classified this as

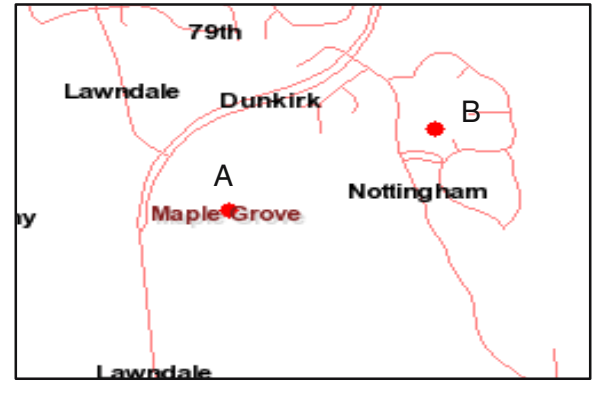

(a)Example of Dot type places

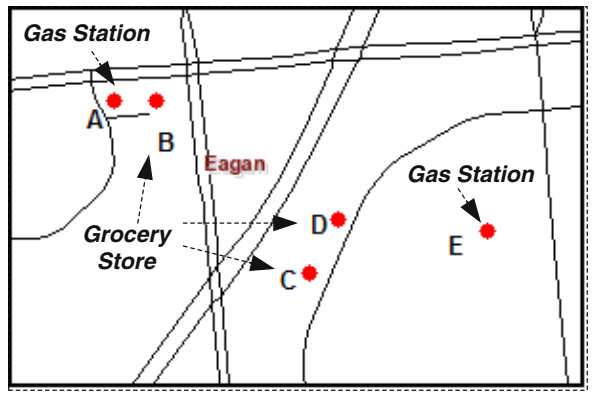

(b)Example of Multiple Dots type places

Fig. 4. Examples of Dot and Multiple Dots type of places: Figure(a) shows a subject's home(A) and friend's house(B); Figure(b) shows a subject's grocery stores(D, C, and $\mathrm{B})$ and gas stations(A and $\mathrm{E})$ 
Dot. Another example is that one subject categorized Dairy Queen (a chain icecream store) as Multiple Dots because she and her daughter stop by more than one or a number of DQs, while she categorized PetMart(a chain pet store) as Dot because she goes only to the one in her neighborhood. Fig. 4(b) shows a section of one subject's map that contains two Multiple Dots places, "grocery store" and "gas station".

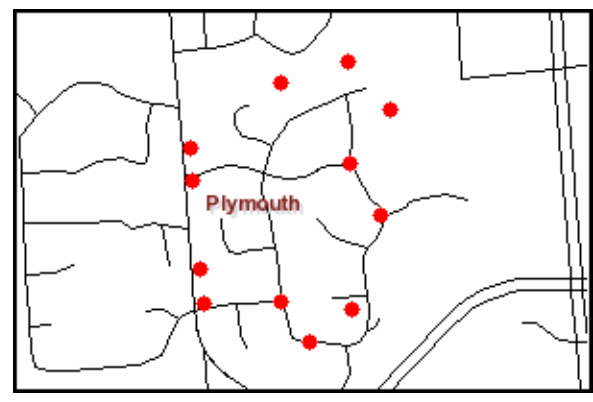

(a)Example of Region type place

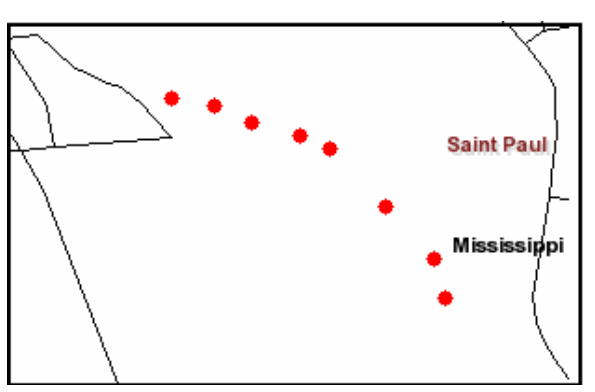

(b)Example of Path type place

Fig. 5. Examples of Region and Path type places: Figure(a) shows a subject's neighborhood region; Figure(b) shows a subject's path for walking her dog in a no-leash dog park by a river

Region. Subjects also made use of the Region concept. Fig. 5(a) shows one subject's region, which consists of his neighborhood. Each of the dots is a specific place contained within the region, e.g., his house, friends' houses, etc.

Some subjects articulated a very interesting point, that is that the very definition of a place depends on the activities one does there. They saw a place as a region if multiple activities took place there, regardless of the size of the place.

"Cooke Hall [campus building] is a Region because that is where I work, teach, talk to my colleagues, and have meetings; MSU [another university] is a Dot because we just drove by and had a quick tour."

"Duluth is a point [Dot] because I just stayed at my son's place; Wal-Mart is a Region because it's got several departments I shop every time."

Note that Regions typically are seen not just as places in their own right but as containers of other places (usually Dots). For example, one subject mentioned that his bank is contained within the (very large) grocery store where he works. Another subject noted that a restaurant he goes to frequently is contained in a hotel some of his friends stayed at.

Path. Over half of the subjects had places of type Path. Typical examples were commuting routes, favorite walks, and bike paths. However, a subject categorized South Dakota(a state in the US) as Path because it represents the 
highway he drives to go camping each summer. One subject categorized Lake Calhoun as a Path because he saw it in terms of the trails around the lake that he rollerbladed on, while other subjects categorized it as Region because they included the parks, theaters and volleyball courts around the lake. Fig. 5(b) shows the path that one subject takes frequently to walk her dog.

\section{Discussion}

The knowledge we have gained of the relationship of places to physical locations leads to design implications and challenges for location-aware systems. Our results identify requirements for the computational representation of places, have implications for systems that aim to acquire place representations, suggest enhanced applications, and open up interesting avenues for future research.

\subsection{Place Representation}

Our results suggest that the places that matter to people take on more complicated physical shapes than prior systems have represented. For some applications to meet their full potential, they must represent these other shapes. The set of shapes we have discussed here can be represented easily by using OpenGIS data structures (www.opengis.org), e.g., as implemented in the PostgreSQL database system. OpenGIS also provides a powerful set of methods, e.g., to find the distance from a point to a region or to find out whether two paths intersect. As we discuss below, we expect these methods to be useful for emerging location-aware applications.

Our interviews also showed that different people may have different conceptions of "the same" place. Some subjects saw Lake Calhoun as a Region, while one saw it as a Path; some subjects think that the grocery chain Cub Foods is Multiple Dots, while others think it is a Dot because they shop at only one Cub Foods location. Our interviews also showed that their conceptions may differ from a "standard" map-based or Geographical Information Systems definition recall that some subjects saw a state (a large geopolitical region in the USA) as a Path, others saw a city as a Dot, and some saw an office building as a Region. These findings highlight the requirement for personal representations of place. The physical geometry of well-known places is a useful resource, but interfaces and acquisition techniques (see below) must allow individual users to create the representations that make sense for them.

\subsection{Place Acquisition}

The more complicated place representations we propose raise challenges for place acquisition techniques. We consider these challenges from three perspectives, suggesting possible courses of action from each perspective.

Algorithms. Existing algorithms [1151311|14|21] are all point-oriented discovering techniques. We need to enhance existing algorithms or develop new 
ones to discover more complicated shapes. Because these shapes contain multiple Dots, knowledge of the associations of individual dots in the group must be extracted. Spatial and temporal data mining techniques, such as motion pattern detection algorithms [7, may be employed to address these problems. Specifically, for Paths, extending existing spatial clustering algorithm to incorporate temporal data processing may be a viable solution. Multiple Dots and Regions could perhaps be discovered by incorporating background knowledge, either of standard places or of places previously acquired for other users (however, recall the caveats about the need for place personalization above). However, we believe that acquisition of the latter two shapes is best done interactively.

Interactive acquisition. It is crucial to give users a role in the acquisition process. This is necessary not just to overcome limits of (even the most advanced) algorithms but also to allow people to express their place concepts as they find easiest. Thus, a discovery algorithm must be embedded in a larger interactive system. A well-designed interface can make it easy for people to indicate that a set of dots really all belong to a Region, or that a sequence of dots really represents a Path. In our work, we are adding interactive capabilities to map-based visualizations like those shown in Fig. 4 and Fig. 5.

Social acquisition. Many places are meaningful not just to a single person but to social groups of varying sizes. In our experiment, we found some students share the same class rooms, study area, lunch places, bus stops and night clubs; some parents share the same community center, city parks, bike trails and grocery stores. Although (as we have noted) one person may not view a particular place the same way as another person, shared places are at least a valuable resource for getting started. For example, suppose Jeff is a student visiting a university campus, and he wants to acquire a set of relevant places to help him in getting around. He might obtain them from his friends who live in the area, for example, a set of restaurants from Joan, some challenging bike routes around campus from Tom, and interesting shopping malls from Jack. Note that collaborative filtering or social matching techniques are useful here, too. That is, even if Jeff doesn't yet know anyone in the area, he could be matched with the people above if (say) he and Joan were both vegetarians, he and Tom were both cyclists, and he and Jack live in the same neighborhoods.

When socially acquiring places, we must note that descriptions of a single place may vary widely, ranging from generic ("a grocery store") to specific ("Cub Foods") to idiosyncratic ("the place we met last time"). Espinoza et al. 5] have provided some anecdotal descriptions of labels people give places when using GeoNotes. Wilenmann and Leuchovius argue that location-based services should describe location in ways relevant to users, such as "I'm home" [20]. In 'Smart Mobs', Rheingold [17] describes a number of applications that will require individuals to share names of their places with others. Consolvo et al. 4 studied factors that impact people's decision to disclose their locations. In our recent study 23], we investigated the types of descriptions people naturally produce for 
places, the extent to which they tailor these descriptions for different audiences, and the factors they consider in deciding how to tailor their descriptions.

\subsection{Opportunities for New Applications}

Existing applications certainly can benefit from this more expressive representation. Consider a virtual notes system, like comMotion or GeoNotes. It is plausible that a user will want to associate her grocery list with any grocery store (Multiple Dots), that her tips for finding a good parking place in the Downtown area should be associated with a broad geographic area (Region), and that people at the beginning of a Path such as a bike route might want to know information about later parts of the path, such as where to get a drink.

New applications may also benefit by incorporating different place shapes. For example, we are interested in the design of navigation aids for visually impaired people. Such users often want to learn routes in a new environment before navigating through it. A user may have certain questions that require complex places to answer, such as: how long is this route? Which routes does this route intersect? How do I get to the emergency exits? What is my relative position in the building?

Different place shapes also empower location-ware social matching applcations to exploit new sharable space and experiences; for example, carpooling applications require representation, identification and matching of drivers' commuting routes (paths).

\section{Summary}

We performed an experiment to investigate how people's concepts of place relate to physical loactions. First, we found that places come in more complex shapes than "points"; in particular the shapes Multiple Dots, Region and Path were common across subjects. Twenty-one percent of all places observed in the study were of these more complex shapes; more than two thirds of the subjects had Multiple Dots and Regions type, and half had Paths. Second, we identified a need for personal representations of palce: different people view places in different ways, and these personal conceptions may not match standard map-based representations.

These findings lead to various implications for the design of location-aware applications. First, place representations must include more complicated shapes. Since people's concepts of place shapes do not always match exactly with the physical shape and people have different concepts for the same place, personal representations and interactive acquisition techniques are required. Second, for a system to acquire more complicated place shapes, algorithms must be improved, interactive techniques must be provided, and social acquisition methods should be explored. Finally, new place shapes and acquisition techniques will empower designers to design new applications in new doamins, such as navigation aids for the visually impaired. 


\section{Acknowledgements}

This work was partially supported by grants from the NSF (IIS 03-07459 and IIS 03-08018). We would like to thank the reviewers for their valuable insights.

\section{References}

1. D. Ashbrook and T. Starner. Learning significant locations and predicting user movement with GPS. In Proc. IEEE Sixth International Symposium on Wearable Computing, 2002.

2. L. Barkhuus and A. Dey. Location-based services for mobile telephony: a study of users privacy concerns. In Proc. Interact, 2003.

3. J. Blom, A. Kankainen, T. Kankainen, and S. Tiitta. Location-aware multiuser messaging: Exploring the evolution of mobile text-based communication services. Technical report, Helsinki Institute for Information Technology (HIIT), 2003.

4. S. Consolvo, I. E. Smith, T. Matthews, A. LaMarca, J. Tabert, and P. Powledge. Location disclosure to social relations: Why, when, \& what people want to share. In Proc. CHI, 2005.

5. F. Espinoza, P. Persson, A. Sandin, H. Nystrm, E. Cacciatore, and M. Bylund. Geonotes: Social and navigational aspects of location-based information systems. In Proc. UbiComp, 2001.

6. K. A. Franck and L. H. Schneekloth, editors. Ordering space: types in architecture and design. Van Nostrand Reinhold, 1994.

7. J. Gudmundsson, M. van Kreveld, and B. Speckmann. Efficient detection of motion patterns in spatio-temporal data sets. In Proc. ACMGIS, 2004.

8. J. Hightower. From position to place. In Proc. Workshop on Location-Aware Computing, 2003.

9. Y. Jung, P. Persson, and J. Blom. Dede: Design and evaluation of a contextenhanced mobile messaging system. In Proc. CHI, pages 351-360, New York, NY, USA, 2005. ACM Press.

10. E. Kaasinen. User needs for location-aware mobile services. Personal and Ubiquitous Computing, 7:70-79, 2003.

11. J. H. Kang, W. Welbourne, B. Stewart, and G. Borriello. Extracting places from traces of locations. In Proc. WMASH, pages 110-118, New York, NY, USA, 2004. ACM Press.

12. P. Klante, J. Krsche, S. Boll, and AccesSights. A multimodal location-aware mobile tourist information system. In Proc. International Conference on Computers Helping People with Special Needs(ICCHP), 2004.

13. L. Liao, D. Fox, and H. Kautz. Learning and inferring transportation routines. In Proc. AAAI, 2004.

14. N. Marmasse and C. Schmandt. Location-aware information delivery with commotion. In Proc. HUC, pages 157-171, 2000.

15. D. Patterson, L. Liao, D. Fox, and H. Kautz. Inferring high-level behavior from low-level sensors. In Proc. UbiComp, 2003.

16. J. S. Pierce and R. Pausch. Navigation with place representations and visible landmarks. In Proc. IEEE VR, 2004.

17. H. Rheingold. Smart Mobs - The Next Social Revolution. Basic Books, 2003.

18. J. Rieman. The diary study: a workplace-oriented research tool to guide laboratory efforts. In Proc. CHI, 1993. 
19. S. Shekhar and S. Chawla. Spatial Databases: A Tour. Prentice Hall (ISBN 07484-0064-6), 2003.

20. A. H. Weilenmann and P. Leuchovius. I'm waiting where we met last time: exploring everyday positioning practices to inform design. In Proc. NordiCHI, 2004.

21. C. Zhou, D. Frankowski, P. Ludford, S. Shekhar, and L. Terveen. Discovering personal gazetteers: An interactive clustering approach. In Proc. ACMGIS, 2004.

22. C. Zhou, P. Ludford, D. Frankowski, and L. Terveen. An experiment in discovering personally meaningful places from location data. In Proc. CHI, Extended Abstract, 2005 .

23. C. Zhou, P. Ludford, D. Frankowski, and L. Terveen. Talking about place: An experiment in how people describe places. In Proc. Pervasive, Short Paper, 2005. 